\title{
Signal Processing Aspects of Cell-Free Massive MIMO
}

\author{
Giovanni Interdonato
}

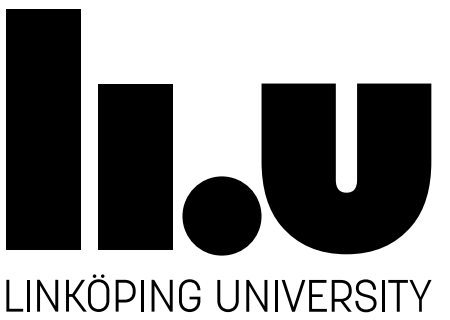

\section{COMMUNICATION SŶsTems}

Division of Communication Systems Department of Electrical Engineering (ISY) Linköping University, 58183 Linköping, Sweden www.commsys.isy.liu.se

Linköping 2018 
This is a Swedish Licentiate Thesis.

The Licentiate degree comprises 120 ECTS credits of postgraduate studies.

Signal Processing Aspects of Cell-Free Massive MIMO

(c) 2018 Giovanni Interdonato, unless otherwise noted.

ISBN 978-91-7685-224-8

ISSN 0280-7971

Printed in Sweden by LiU-Tryck, Linköping 2018 


\section{Abstract}

The fifth generation of mobile communication systems (5G) promises unprecedented levels of connectivity and quality of service (QoS) to satisfy the incessant growth in the number of mobile smart devices and the huge increase in data demand. One of the primary ways $5 \mathrm{G}$ network technology will be accomplished is through network densification, namely increasing the number of antennas per site and deploying smaller and smaller cells.

Massive MIMO, where MIMO stands for multiple-input multiple-output, is widely expected to be a key enabler of $5 \mathrm{G}$. This technology leverages an aggressive spatial multiplexing, from using a large number of transmitting/receiving antennas, to multiply the capacity of a wireless channel. A massive MIMO base station (BS) is equipped with a large number of antennas, much larger than the number of active users. The users are coherently served by all the antennas, in the same time-frequency resources but separated in the spatial domain by receiving very directive signals. By supporting such a highly spatially-focused transmission (precoding), massive MIMO provides higher spectral and energy efficiency, and reduces the inter-cell interference compared to existing mobile systems. The inter-cell interference is however becoming the major bottleneck as we densify the networks. It cannot be removed as long as we rely on a network-centric implementation, since the inter-cell interference concept is inherent to the cellular paradigm.

Cell-free massive MIMO refers to a massive MIMO system where the BS antennas, herein referred to as access points (APs), are geographically spread out. The APs are connected, through a fronthaul network, to a central processing unit (CPU) which is responsible for coordinating the coherent joint transmission. Such a distributed architecture provides additional macro-diversity, and the co-processing at multiple APs entirely suppresses the inter-cell interference. Each user is surrounded by serving APs and experiences no cell boundaries. This user-centric approach, combined with the system scalability that characterizes the massive MIMO design, constitutes a paradigm shift compared to the conventional centralized and distributed 
wireless communication systems. On the other hand, such a distributed system requires higher capacity of back/front-haul connections, and the signal co-processing increases the signaling overhead.

In this thesis, we focus on some signal processing aspects of cell-free massive MIMO. More specifically, we firstly investigate if the downlink channel estimation, via downlink pilots, brings gains to cell-free massive MIMO or the statistical channel state information (CSI) knowledge at the users is enough to reliably perform data decoding, as in conventional co-located massive MIMO. Allocating downlink pilots is costly resource-wise, thus we also propose resource saving-oriented strategies for downlink pilot assignment. Secondly, we study further fully distributed and scalable precoding schemes in order to outperform cell-free massive MIMO in its canonical form, which consists in single-antenna APs implementing conjugate beamforming (also known as maximum ratio transmission). 


\section{Populärvetenskaplig sammanfattning}

Den femte generationen mobilkommunikationssystem (5G) utlovar tidigare oöverträffade nivåer av servicekvalitet (QoS) ämnat att möjliggöra en fortsatt tillväxt i både antalet mobila smarta enheter och dataanvändning. Ett av de primära sätten detta kommer att uppnås är genom nätverksförtätning, dvs. att använda mindre och mindre celler samt att öka antalet antenner per cell.

Massiv MIMO (där MIMO står för multiple-input multiple-output) förväntas utgöra en nyckelkomponent i 5G. Denna teknik möjliggör en aggressiv spatial multiplexering av datasignaler genom att använda ett stort antal sändande och/eller mottagande antenner som multiplicerar kapaciteten i radiokanalen. En massiv MIMO basstation är utrustad med ett stort antal antenner, mycket större än antalet aktiva användare. Användarna betjänas faskoherent och i samma tids- och frekvensresurser av alla antennerna, och deras signaler separeras i den rumsliga domänen. Genom att stödja en sådan mycket rumsfokuserad överföring ger massiv MIMO högre spektral- och energieffektivitet, samt minskar intercellinterferensen jämfört med befintliga mobilsystem. Intercellinterferensen utgör dock en fortsatt stor flaskhals när vi förtätar nätverken och kan inte avlägsnas helt i ett nätverkscentrerat och cellulärt paradigm.

Cellfri massiv MIMO refererar till ett massivt MIMO-system där basstationsantennerna, häri refererade till som accesspunkter (AP), sprids geografiskt. AP-enheterna är anslutna till en central processor (CPU, central processing unit) som ansvarar för att samordna den gemensamma faskoherenta överföringen. En sådan distribuerad nätverksarkitektur skapar mycket hög makrodiversitet, och central processande av signaler från flera AP-enheter möjliggör nära nog fullständig undertryckning av intercellinterferensen i systemet. Varje användare är omgiven av aktiva AP-enheter och upplever inga cellgränser. Detta användarcentrerade tillvägagångssätt kombinerat med systemskalbarheten som kännetecknar den massiva MIMO-designen, utgör ett 
paradigmskifte jämfört med konventionella centraliserade och distribuerade trådlösa kommunikationssystem. Å andra sidan ställs det i ett sådant distribuerat system högre kapacitetskrav för anslutningar till-och-från AP-enheterna och CPU-enheten (backhaul/fronthaul) och det centrala signalprocessandet ökar kostnaden för signalering.

I denna licentiatavhandling fokuserar vi på några signalbehandlingsaspekter av cellfri massiv MIMO. Mer specifikt undersöker vi om kanalsuppskattning med hjälp av nedlänkspiloter ger vinster till cellfri massiv MIMO eller om statistisk kanalinformation (CSI, channel state information) hos användarna är tillräckligt för att på ett tillförlitligt sätt utföra avkodning av data. Att fördela nedlänkspiloter till alla användare är kostsamt, så vi föreslår också resursbesparingsinriktade strategier för nedlänkspilotilldelning. Vidare studerar vi fullständigt distribuerade och skalbara förkodningssystem som överträffar cellfri massiv MIMO i sin kanoniska form. 


\section{Acknowledgments}

First of all, I have to thank Prof. Erik G. Larsson and Dr. Gunnar Bark, for giving me the opportunity to join the Division of Communication Systems and Ericsson Research, respectively. It makes me feel very proud and honored pursuing the Ph.D. degree in these prestigious workplaces, closely to such a brilliant people.

I owe my deepest gratitude to my supervisors Prof. Erik G. Larsson, Dr. Pål Frenger and Dr. Hien Quoc Ngo for the continuous support of my Ph.D. study and research, their motivation, enthusiasm and optimism, expertise and immense knowledge. Everything becomes easier thanks to their encouragement and support. Without their guidance and persistent help this thesis would not have been possible.

I would like to express my sincere gratitude to my former manager Dr. Gunnar Bark and my current manager Dr. Nicklas Johansson for their keen interest, affection and care shown towards me during my stay at Ericsson. Their interest and involvement in my research and concern for my welfare have greatly motivated me.

Special thanks also to Associate Professor Emil Björnson for giving me insightful comments, constructive feedback and suggestions whenever I need.

I am grateful to all my colleagues and friends at LINLAB and the Communication Systems division for the time spent together. All of them helped me grow both personally and professionally.

I would also like to express my gratitude to the "5Gwireless" project (H2020 Marie Skłodowska-Curie Innovative Training Networks) for its financial support. My warm thanks to Prof. Marco Di Renzo for its hard work as coordinator of the $5 \mathrm{Gwireless}$ project, and its assistance during all the project activities. My heartiest thanks to my 5Gwireless colleagues for all the time we shared in the last three years. We worked hard and played hard. I wish them all the best for the future.

I have no words to express my gratitude for my parents for their love, prayer, support, care and encouraging words that made me the man I am. 
Last but foremost, I dedicate this thesis to my love, Silvia. You are the strength that keeps me walking.

Giovanni Interdonato Linköping, August 2018 


\section{Contents}

$\begin{array}{ll}\text { Introduction } & 1\end{array}$

1 Cell-Free Massive MIMO 3

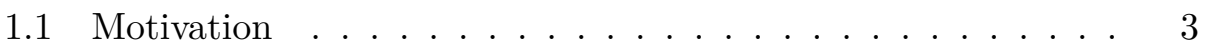

1.2 The Paradigm Shift . . . . . . . . . . . . . 3

1.2.1 The Scalable Way to Implement CoMP-JT . . . . . . 6

1.2.2 TDD Protocol . . . . . . . . . . . . . . 9

1.3 Practical Deployment Issues . . . . . . . . . . . . . . . . . 11

1.3.1 Radio Stripe System . . . . . . . . . . . . . . . . 11

1.3.2 Front-haul and Back-haul Capacity . . . . . . . . . . 13

1.3.3 Synchronization . . . . . . . . . . . . . . . . 14

1.3.4 Power Control . . . . . . . . . . . . . . . . 16

1.3.5 Pilot Assignment . . . . . . . . . . . . . . . 18

1.4 Performance of "cell-free" Massive MIMO . . . . . . . . . . . 18

1.4 .1 Industrial Indoor Scenario . . . . . . . . . . . . . . . 19

1.4.2 Piazza Scenario . . . . . . . . . . . . . . . . 22

1.5 Research Directions . . . . . . . . . . . . . . 22

2 Contributions of the Thesis $\quad 25$

2.1 Introduction of the Thesis . . . . . . . . . . . 25

2.2 Papers Included in the Thesis . . . . . . . . . . . . . 26

2.3 Papers Not Included in the Thesis . . . . . . . . . . . . 28

$\begin{array}{lll}3 & \text { Conclusions } & 29\end{array}$

4 Future Work $\quad 31$

$\begin{array}{ll}\text { Bibliography } & 33\end{array}$ 
A Downlink Training in Cell-Free Massive MIMO 39

1 Introduction . . . . . . . . . . . . . . . 41

2 System Model . . . . . . . . . . . . . . . . . . . . 44

2.1 Uplink Training and Channel Estimation . . . . . . 46

2.2 Downlink Data Transmission . . . . . . . . . . . 48

2.3 Downlink Training and Channel Estimation . . . . . . 49

3 Channel Hardening in Cell-Free Massive MIMO . . . . . . . . 51

4 Performance Analysis . . . . . . . . . . . . . . 53

$4.1 \quad$ Approximate Achievable Downlink Rate . . . . . . . . 53

4.2 Use-and-Forget Achievable Downlink Rate . . . . . . . 57

4.3 Downlink Pilots, or No Downlink Pilots, that is the

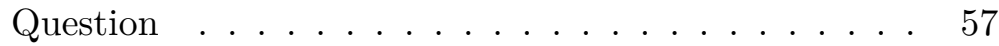

5 Resource Allocation . . . . . . . . . . . . . . . 59

5.1 Max-Min Fairness Power Control . . . . . . . . . 59

5.2 Pilot Sequence Assignment . . . . . . . . . . . . . 64

6 Numerical Results and Discussions . . . . . . . . . . . . . 66

6.1 Simulation Scenario . . . . . . . . . . . . . 66

6.2 Pilot Allocation . . . . . . . . . . . . . . 68

6.3 Performance of the SCA Algorithm for Max-Min Power

Control . . . . . . . . . . . . . . . 71

6.4 Downlink Training Gain . . . . . . . . . . . . . 72

6.5 Downlink Training Gain under Max-Min Power Control 74

6.6 Downlink Training Gain in User-centric Massive MIMO

Networks . . . . . . . . . . . . . 76

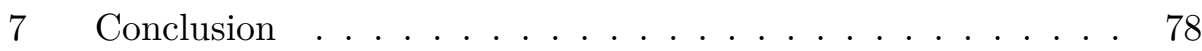

$8 \quad$ Appendix . . . . . . . . . . . . . . . . 79

$8.1 \quad$ Proof of Proposition $1 \ldots \ldots \ldots 79$

8.2 Proof of Proposition $2 \ldots \ldots \ldots$. . . . . . . . 82

8.3 Proof of Proposition $3 \ldots \ldots \ldots$

References...................... 83

B Downlink Pilot Assignment in Cell-Free Massive MIMO $\quad \mathbf{8 7}$

1 Introduction . . . . . . . . . . . . . . . . 89

2 Pilot Assignment in Massive MIMO _. . . . . . . . . . . . 91

2.1 TDD Protocol and Resource Allocation . . . . . . . . 91

2.2 Pilot Contamination and Orthogonal Pilot Transmission 92

3 System Model . . . . . . . . . . . . . . . . . . . . 94

4 Achievable Downlink Rate . . . . . . . . . . . . . . . 96 
4.1 Fading Channel with Statistical CSI at the Receiver . 96

$4.2 \quad$ Fading Channel with Side Information . . . . . . . 97

5 Utility-based DL Pilot Assignment . . . . . . . . . . . . . 97

$5.1 \quad$ Proposed Scheme . . . . . . . . . . . . . . 97

$5.2 \quad$ Pilot Utility Metric . . . . . . . . . . . . . . . . . . . 99

6 Numerical Results . . . . . . . . . . . . . . . . . . . . . . 101

6.1 Simulation Scenario . . . . . . . . . . . . . . . . 101

6.2 Performance Evaluation . . . . . . . . . . . . 102

7 Conclusion . . . . . . . . . . . . . . 107

References . . . . . . . . . . . . . . . 107

C Cell-Free Massive MIMO with Short-Term Power Con$\begin{array}{ll}\text { straints } & 109\end{array}$

1 Introduction . . . . . . . . . . . . . . . . . . . 111

2 System Model and Notation . . . . . . . . . . . . . . . 112

2.1 Uplink Training and Channel Estimation . . . . . . 113

2.2 Downlink Data Transmission . . . . . . . . . . . . . 114

3 Performance Analysis . . . . . . . . . . . . . . . 115

4 Numerical Results . . . . . . . . . . . . . . . . . . 117

$4.1 \quad$ Simulation Scenario and Parameters . . . . . . . . 118

4.2 Performance Evaluation . . . . . . . . . . . . . . . . 119

5 Conclusion and Future Work . . . . . . . . . . . . . . 120

6 Appendix . . . . . . . . . . . . . . . . . 122

6.1 Proof of Proposition $1 \ldots \ldots$. . . . . . . . 122

References . . . . . . . . . . . . . . . . . . 124

D Cell-Free Massive MIMO with Full-Pilot Zero-Forcing $\quad 127$

1 Introduction . . . . . . . . . . . . . . . . . . . 129

2 System Model . . . . . . . . . . . . . . . . . . . 130

2.1 Uplink Training and Channel Estimation . . . . . . 131

2.2 Downlink Data Transmission . . . . . . . . . . . . 132

3 Performance Analysis . . . . . . . . . . . . . . . . . 133

$3.1 \quad$ Achievable Downlink Spectral Efficiency . . . . . . . 133

3.2 Achievable Downlink Spectral Efficiency for Indepen-

dent Rayleigh Fading and Full-Pilot Zero-Forcing . . . 134

3.3 Max-Min Fairness Power Control . . . . . . . . . . . . 134

4 Numerical Results . . . . . . . . . . . . . . . . . 136

4.1 Performance Evaluation . . . . . . . . . . . . 136

5 Conclusion . . . . . . . . . . . . . . . . . 139

References . . . . . . . . . . . . . . . . 139 



\section{Introduction}





\section{Chapter 1}

\section{Cell-Free Massive MIMO}

\subsection{Motivation}

The concept of delivering widespread wireless communication services by splitting a coverage area into many cells dates back to the 1940s and the first practical cellular networks were trialled in the 1970s [1]. Since then, we have witnessed an incredible wireless revolution, where cellular connectivity has gone from being a luxury product to a commodity and where the main application has changed from voice calls to data transfer. From $1 \mathrm{G}$ to $4 \mathrm{G}$, the massive traffic growth has been managed by a combination of wider bandwidths, refined radio interfaces, and smaller cells [2]. Due its costefficiency, the latter has contributed the most; cell towers used to be $10 \mathrm{~km}$ apart and are now only $100 \mathrm{~m}$ apart in urban areas. While rigid frequency planning was originally used to alleviate inter-cell interference, the interference is becoming a major bottleneck as we densify the networks [3], because each cell is then hit by non-negligible interference from many neighboring cells. One way to deal with inter-cell interference is to manage it spatially by Massive MIMO (multiple-input multiple-output) methods [4,5], which is a key 5G technology [6]. However, looking further ahead, we need a more radical approach; we need to challenge the cellular paradigm and realize that inter-cell interference is not a fundamental phenomenon but the result of the cell boundaries that we have purposely created in the data transmission.

\subsection{The Paradigm Shift}

A conventional cellular network is characterized by the fact that each user equipment (UE) is connected to the access point (AP) in only one of the many cells (except during handover); see Fig. 1a. This is a practically convenient 
assumption since each uplink/downlink data signal is only processed by a single AP. At a given time instance, the APs have different number of active UEs and the transmissions in one cell cause interference to the UEs in other cells. Since a typical AP is equipped with 1-4 antennas, its ability to mitigate interference spatially, by beamforming, is rather limited. Cellular networks are suboptimal from a channel capacity viewpoint because much higher spectral efficiency (bit/s/Hz/user) can be achieved by allowing for co-processing at multiple APs [7]. The co-processing approach is known under many names, including distributed wireless communication system [3], network MIMO [8,9], coordinated multipoint with joint transmission (CoMP-JT) [10,11], and multicell MIMO cooperative networks [12]. The conventional network-centric way to implement this is to divide the APs into disjoint clusters, as illustrated in Fig. 1b. The APs in a cluster transmit jointly to the UEs residing in their joint coverage area. In principle, this is no different than deploying a conventional cellular network with distributed antennas in each cell. Hence, despite the great theoretical gains, the 3GPP LTE standardization of CoMPJT has not provided any remarkable practical gains [11]. This fact does not mean that the basic concept is flawed, but the network-centric approach may not be preferable.

Next, we describe a new concept that takes CoMP-JT to its practically implementable level. To avoid preconceptions, we use the new "cell-free" communication terminology from $[13,14]$ instead of prior terminology. The word "cell-free" signifies that, at least from a user perspective, there are no cell boundaries during data transmission, but all APs in the network cooperate to jointly serve the users in a user-centric fashion. For a given user, the closest APs are involved in the data transmission, while all APs that affect the user take the interference that they cause into consideration. This principle is illustrated in Fig. 1c. In contrast to the cell boundaries created by the network-centric approach, the user-centric approach gives full control over the interference. That said, we stress that also in a "cell-free" network, it is convenient to have AP-specific synchronization and reference signals, which are important when accessing the network. These signals can be viewed as cell identifiers, thus "cell-free" means that there are no cell boundaries created by the data transmission protocol in active mode.

A basic "cell-free" network is illustrated in Fig. 1d. The APs are connected via front-haul connections to central processing units (CPUs). If there are multiple CPUs in the deployment area, then the CPU are connected by back-haul links to enable user-centric communication also in areas covered by several CPUs.

Before covering the conceptual details, we will illustrate the paradigm 


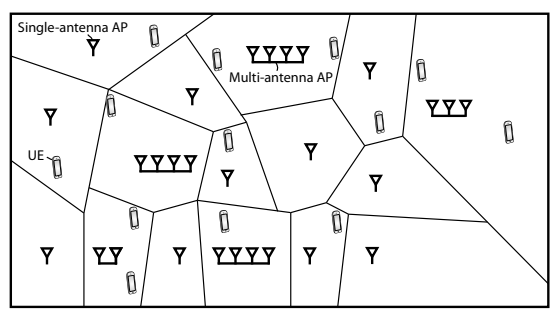

(a) A conventional cellular network where each UE is connected to only one AP.

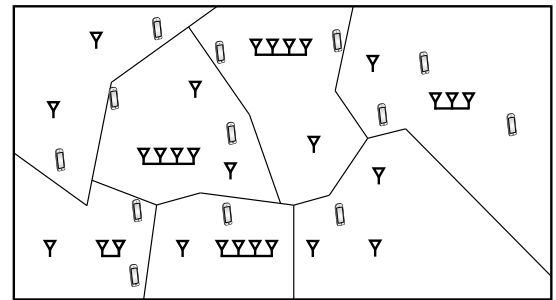

(b) A conventional network-centric implementation of CoMP-JT, where the APs form disjoint clusters that act as cells in the sense that the APs in a cluster communicate with the UEs residing in their joint coverage area.

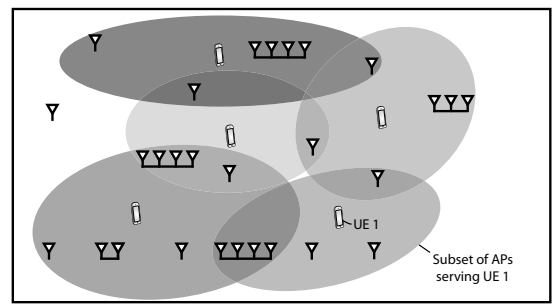

(c) In user-centric networks, each UE communicates with a user-specific subset of the APs and hence there are no cell boundaries and no inter-cell interference in the data transmission.

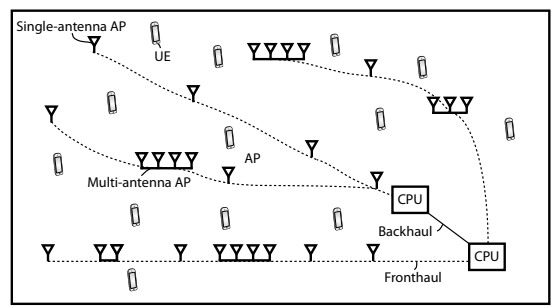

(d) A "cell-free" network is a way to implement a user-centric network.

Figure 1: With the same collection of APs, we can create many different types of communication networks. 
shift that "cell-free" networks will constitute, particularly in terms of celledge performance. Fig. 2 shows the performance that is achieved at different locations in an area where nine APs are deployed in a regular pattern: (a) shows that the spectral efficiencies in a cellular network are poor at the cell edges due to strong inter-cell interference (irrespective of the cell sizes) while (b) shows that a "cell-free" network can avoid interference by co-processing over the APs and only suffers from the physical signal propagation path losses from the APs. Since cell-center UEs obtain higher data rates than cell-edge UEs, the latter need to be active for a longer time to receive a data packet of a certain size; thus, at a given time instance, most of the active UEs are likely at the cell edge. The fact that "cell-free" networks provide the largest performance gain to these UEs demonstrate how effectively it can alleviate inter-cell interference [13].

\subsubsection{The Scalable Way to Implement CoMP-JT}

The first challenge in implementing a "cell-free" network is to obtain sufficiently accurate channel state information (CSI) so that the APs can simultaneously transmit (receive) signals to (from) all UEs and cancel interference in the spatial domain. This principle is similar to beamforming, but the downlink signals will not form beams since the transmitters are distributed. The conventional approach of sending a collection of downlink pilot signals and letting the UEs feed back estimates of the downlink channels is unscalable since the feedback load is proportional to the number of APs. The path to circumvent this scalability issue starts with the observation in [15] that each AP only requires local CSI to perform its tasks, which refers to the channel between the AP and each of the UEs. Local CSI can be conveniently acquired in time-division duplex (TDD) operation since, when a UE sends a pilot sequence, each AP can simultaneously estimate its channel to the UE. Hence, the channel estimation overhead is independent of the number of APs. By exploiting channel reciprocity, the uplink channel estimates can be also utilized as downlink channel estimates. This is basically the same approach to CSI acquisition as in conventional cellular Massive MIMO [5].

Inspired by the fact that Massive MIMO is the scalable way to implement multi-user MIMO, a series of recent works have considered ubiquitous "cellfree" Massive MIMO as the scalable way to implement CoMP-JT $[13,14,16]$. This refers to a "cell-free" network with a massive number $L$ of geographically distributed APs that jointly serve a relatively smaller number $K$ of users: $L \gg K$. These networks provide substantially better 95\%-likely spectral efficiency for the UEs than a corresponding cellular network with small cells; 


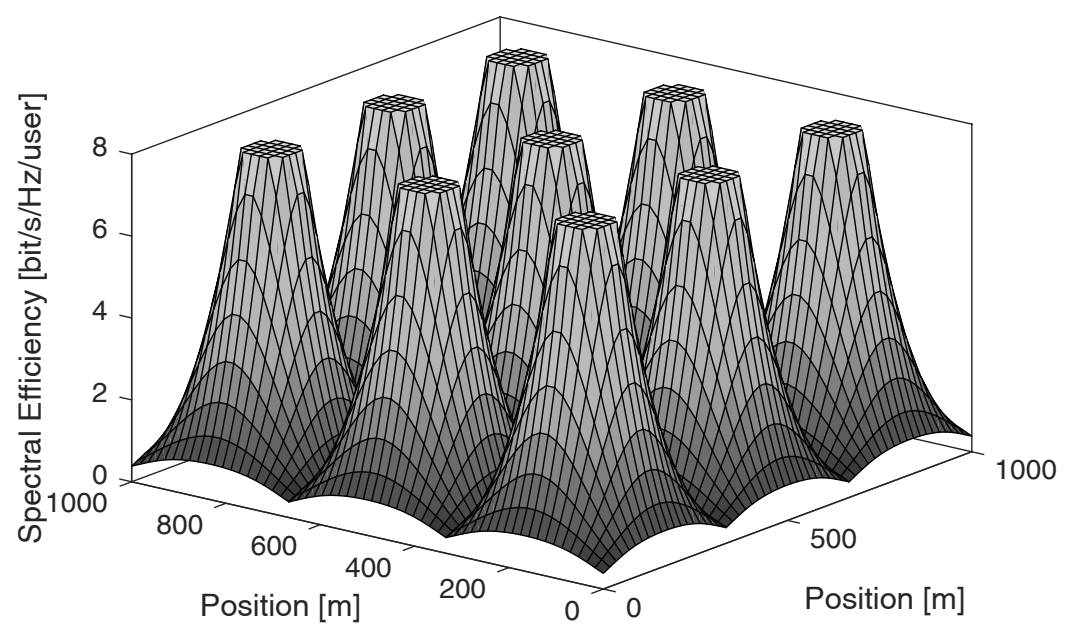

(a) Data coverage in a cellular network.

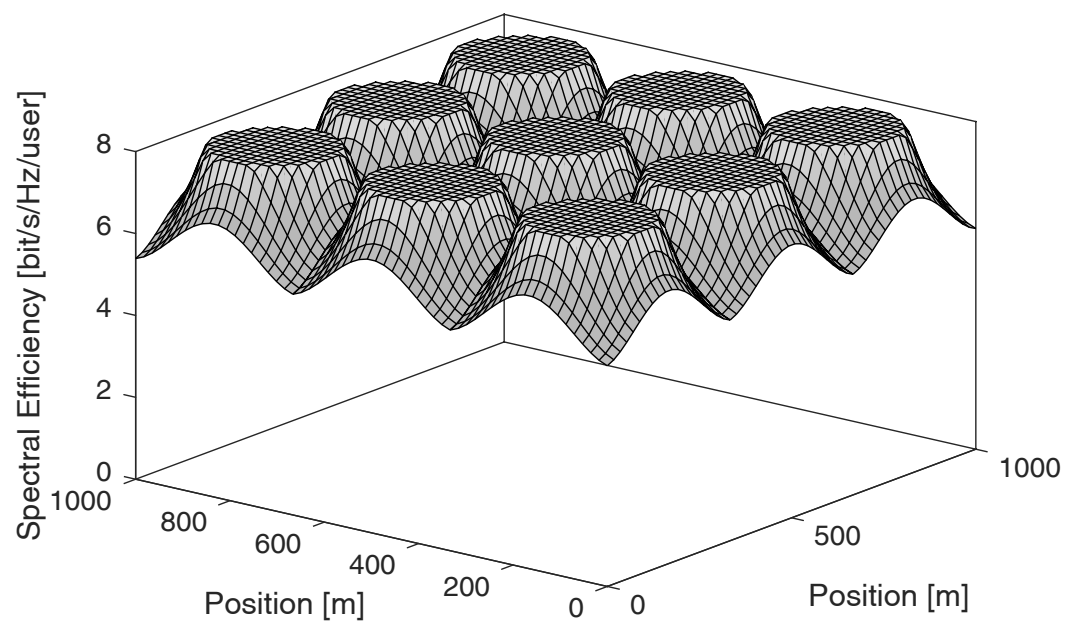

(b) Data coverage in a "cell-free" network.

Figure 2: Spectral efficiency achieved by UEs at different locations. The cellular network in (a) suffers from poor cell-edge throughput due to inter-cell interference, while the "cell-free" network in (b) is only limited by signal propagation losses. Note that $8 \mathrm{bit} / \mathrm{s} / \mathrm{Hz}$ was selected as the maximal spectral efficiency, which corresponds to uncoded 256-QAM.

the simulations in $[13,14]$ shows 5 -10 fold improvements.

There are two key properties behind this result. The first property is the additional macro-diversity provided by the fact that each UE receives signals 


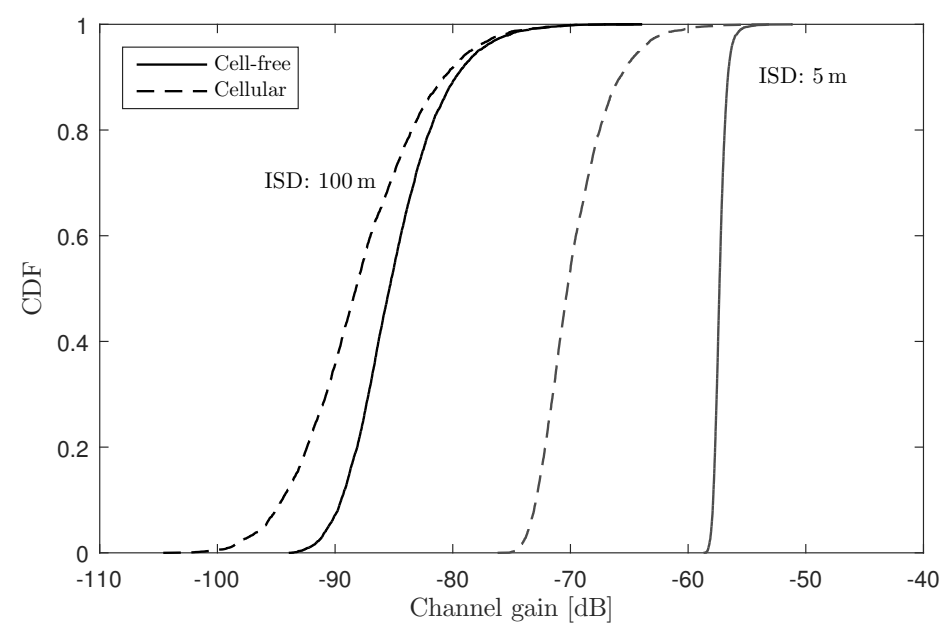

(a) Distribution of the channel gain.

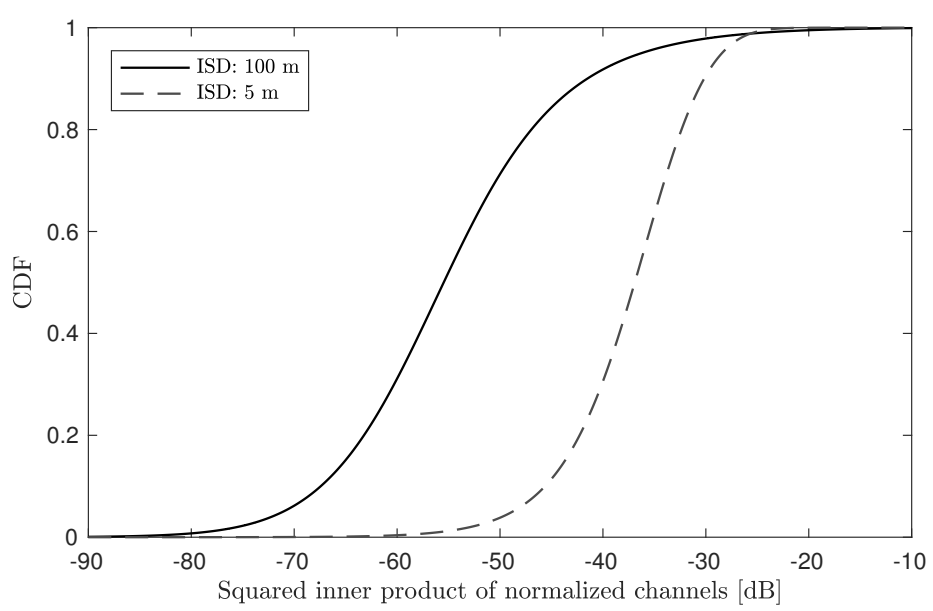

(b) Distribution of the inner product of channel vectors.

Figure 3: Two key properties that make "cell-free" Massive MIMO desirable. (a) The distribution of the channel gain is more beneficial than in a cellular system, thanks to the additional macro-diversity; (b) The inner product of the channel vectors of two randomly selected UEs is very small, which is called favorable propagation. The simulation setup considers 2500 singleantenna APs deployed on a square grid with wrap-around and varying ISD. The small-scale fading is modeled by independent Rayleigh fading and the large-scale fading is computed using the three-slope model from [13] (see Section 6.1 of Paper A for the details). 
from multiple APs. Fig. 3a illustrates this by considering a setup with 2500 single-antenna APs deployed on a square grid with wrap-around and varying inter-site distance (ISD): 5 and $100 \mathrm{~m}$. The figure shows the cumulative distribution function (CDF) of the channel gain for a UE at a random position in the network with channel vector $\mathbf{h}=\left[h_{1} \ldots h_{L}\right]^{\mathrm{T}} \in \mathbb{C}^{L}$, where $h_{l}$ is the channel from the $l$ th AP. The channel gain is $\|\mathbf{h}\|^{2}$ in "cell-free" Massive MIMO and $\max _{l}\left|h_{l}\right|^{2}$ in a cellular network. With a large ISD, the UEs with the best channel conditions have almost identical channel gains in both cases, but the most unfortunate UEs gains $5 \mathrm{~dB}$ from "cell-free" processing. With a small ISD of $5 \mathrm{~m}$, which is reasonable for connected factory applications, all UEs obtain 5-20 dB higher channel gain by the "cell-free" network.

The second property is known as favorable propagation and means that the channel vectors $\mathbf{h}_{1}, \mathbf{h}_{2}$ of any pair of UEs are nearly orthogonal and thus the APs can efficiently suppress interference between the UEs. This phenomenon appears in cellular networks with Massive MIMO [5] since the small-scale fading is likely to make the large-dimensional channel vectors pairwise orthogonal. The level of orthogonality can be measured by

$$
\frac{\left|\mathbf{h}_{1}^{\mathrm{H}} \mathbf{h}_{2}\right|^{2}}{\left\|\mathbf{h}_{1}\right\|^{2}\left\|\mathbf{h}_{2}\right\|^{2}},
$$

which is the squared inner product of the normalized channels. A smaller value represents greater orthogonality. In a cellular network with singleantenna APs, $\mathbf{h}_{1}$ and $\mathbf{h}_{2}$ are scalars and thus the measure is always one. Favorable propagation will, however, appear in "cell-free" Massive MIMO where $\mathbf{h}_{1}, \mathbf{h}_{2} \in \mathbb{C}^{L}$, since the combination of small-scale and large-scale fading makes the large-dimensional channel vectors pairwise nearly orthogonal [16]. This is illustrated in Fig. 3b, which shows the CDF of the orthogonality measure for two randomly located UEs. The inner product is very small for all the considered ISDs. Note that it increases as the ISD reduces, since the pathloss exponent is smaller when the distances are short.

\subsubsection{TDD Protocol}

The TDD protocol recommended for "cell-free" Massive MIMO is illustrated in Fig. 4. Each AP estimates the uplink (UL) channel from each UE by measurements on UL pilots. By virtue of reciprocity, these estimates are also valid for the DL channels. Hence, the pilot resource requirement is independent of the number of AP antennas and no UL feedback is needed.

After applying precoding, each UE sees an effective scalar channel. The UE needs to estimate the gain of this channel to decode its data. Note that 


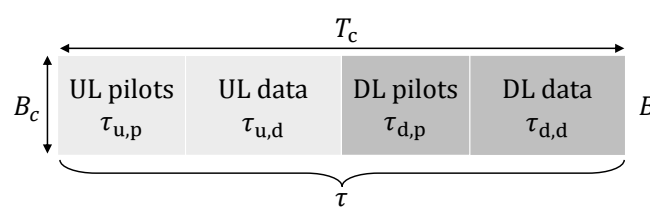

(a) TDD frame with DL training

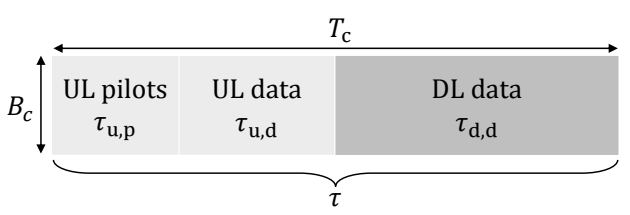

(b) TDD frame without DL training

Figure 4: The TDD frame is $\tau=T_{\mathrm{c}} B_{\mathrm{c}}$ symbols long, where $B_{\mathrm{c}}$ and $T_{\mathrm{c}}$ are the coherence bandwidth and the coherence time, respectively. In practice, there are also guard intervals, but these are deducted from the coherence time interval in this frame structure. The DL channel estimation can either be implemented using explicit DL pilots as in (a) or by estimating the effective DL scalar from the DL data transmission as in (b). In the latter case, no DL pilots are needed and there is more space for data. Note that the latter is used in cellular Massive MIMO, which can rely on channel hardening, while both options are on the table for "cell-free" Massive MIMO.

in cellular Massive MIMO, owing to channel hardening, the UE may rely on knowledge of the average channel gain for decoding [5]. In "cell-free" Massive MIMO, in contrast, there is less hardening and DL effective gain estimation is desirable at the terminal $[16,17]$. This estimate can either be obtained from DL pilots sent by the AP during a DL training phase [17] or, potentially, blindly from the DL data transmission if there are no DL pilots; see Fig. 4.

The channel coherence interval is defined as the time-frequency interval during which the channel can be approximately considered as static. It is determined by the propagation environment, UE mobility, and carrier frequency [5]. The TDD frame should be shorter than the smallest coherence time among the active UEs. For simplicity, we herein treat the TDD frame and coherence interval as interchangeable terms.

Fig. 4 shows two possible frame configurations, with and without DL training. The frame including the DL training phase, depicted in Fig. 4a, consists of: ( $i$ ) UL training phase (UL pilots); (ii) UL data transmission (UL data); (iii) DL training phase (DL pilots); and (iv) DL data transmission (DL data). Fig. 4b illustrates the TDD frame without DL training.

Let $\tau=T_{\mathrm{c}} B_{\mathrm{c}}$ the length of the coherence interval in symbols, where $B_{\mathrm{c}}$ is the coherence bandwidth and $T_{\mathrm{c}}$ indicates the coherence time. Let $\tau_{\mathrm{p}}=\tau_{\mathrm{u}, \mathrm{p}}+\tau_{\mathrm{d}, \mathrm{p}}$ denote the total number of symbols per coherence interval spent on transmission of UL and DL pilots, $\tau_{\mathrm{d}, \mathrm{d}}$ and $\tau_{\mathrm{u}, \mathrm{d}}$ denote the number of symbols per coherence interval spent on transmission of DL and UL data, respectively. The length of the TDD frame is given by $\tau=\tau_{\mathrm{p}}+\tau_{\mathrm{d}, \mathrm{d}}+\tau_{\mathrm{u}, \mathrm{d}}$. 
The maximum number of mutually orthogonal pilots is upper-bounded by $\tau$. Hence, allocating orthogonal pilots is physically impossible in networks with $K \geq \tau$, and it necessary adopting either pilot reuse or non-orthogonal pilots. UEs that send the same pilot cause mutual interference that make the respective channel estimates correlated, a phenomenon known as pilot contamination [18]. This results in a degradation of the channel estimation quality and in coherent interference.

\subsection{Practical Deployment Issues}

The cost and complexity of deployment, limited capacity of back/front-haul connections, and network synchronization are three major issues that need be solved in a practical deployment.

\subsubsection{Radio Stripe System}

The cabling and internal communication between antennas is challenging in practical "cell-free" deployments. A star topology with a separate cable between each antenna and a CPU may be economically infeasible. An appropriate, cost-efficient architecture is the radio stripe system [19], presented next.

In a radio stripe system, the antennas and the associated antenna processing units (APUs) are serially located inside the same cable, which also provides synchronization, data transfer, and power supply via a shared bus; see Fig. 5a. More specifically, the actual APs consist of circuit-mounted chips inside the protective casing of a cable or a stripe. Each radio stripe is then connected to one or multiple CPUs. The receive/transmit processing of an antenna is performed right next to itself. Since the total number of distributed antennas is assumed to be large, the transmit power of each antenna can be very low, resulting in low heat-dissipation, small volume and weight, and low cost.

Small low-gain antennas are used. For example, if the carrier frequency is $5.2 \mathrm{GHz}$ then the $\lambda / 2$ antenna size is $2.8 \mathrm{~cm}$, thus, the antennas and processing hardware can be easily fitted in a cable/stripe. This cable contains antennas, power amplifiers, phase shifters, filters, modulators, A/D and D/A converters.

On the transmitter side, each APU receives up to $K$ streams of input data (e.g., one stream per UE, one UE with $K$ streams, or some other UE-stream allocation) from the previous APU via the shared bus. In each antenna, the input data streams are scaled with the pre-calculated precoding vector and the sum-signal is transmitted over the radio channel to the receiver(s). By 


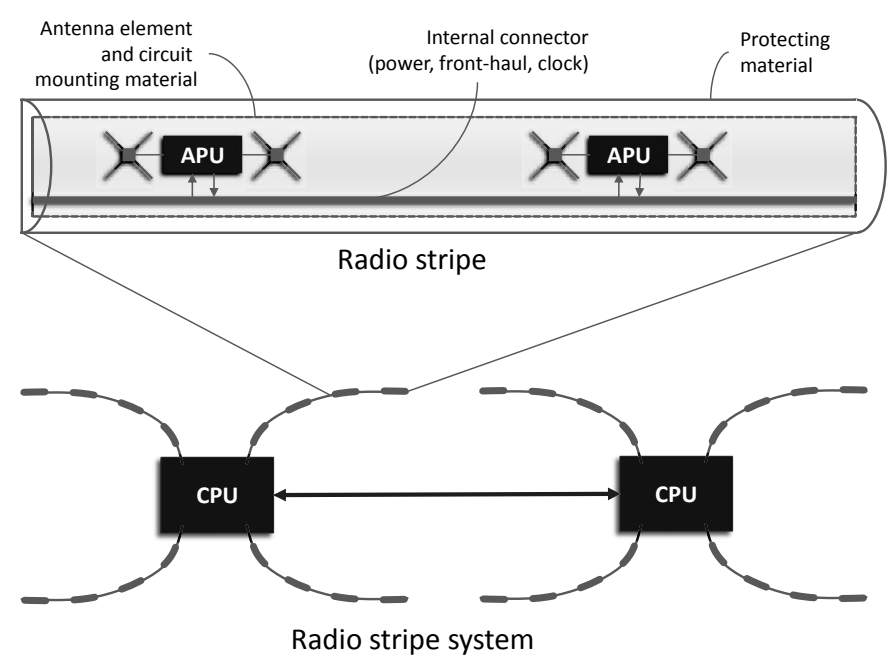

(a) Example of a radio stripe system.
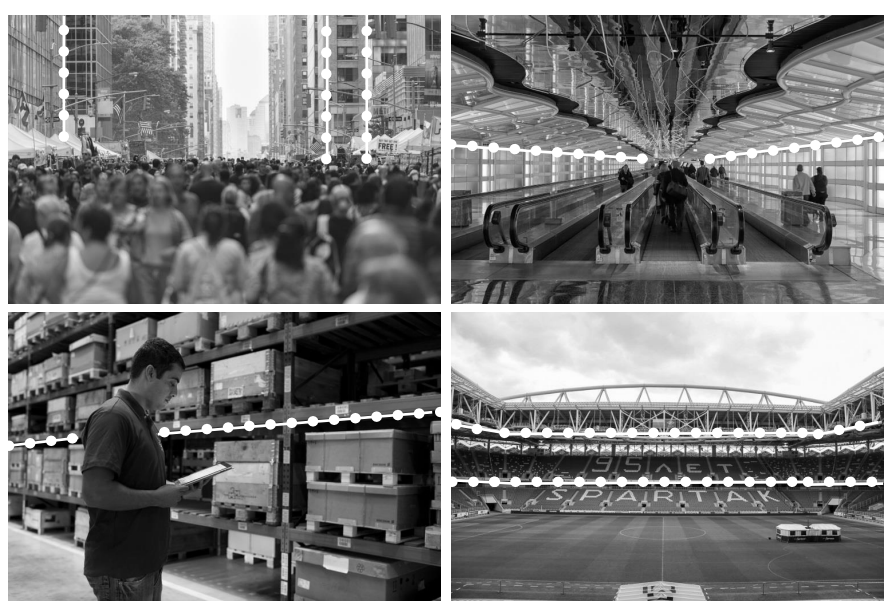

(b) Radio stripes, here illustrated with solid lines and dots, enable invisible installation in existing construction elements.

Figure 5: The radio stripe system concept leads to very flexible deployment of "cell-free" Massive MIMO networks. The basic building blocks are shown in (a), while different deployment concepts are exemplified in (b). The total length of a single radio stripe may vary, e.g., from 10 to 100 meters. Each radio stripe sends/receives data to/from one or multiple CPUs through a shared bus (or internal connector), which also provides synchronization and power supply to each APU. 
exploiting channel reciprocity, the precoding vector may be a function of the estimated uplink channels. For example, if the conjugate of the estimated uplink channel is used, maximum ratio (MR) precoding (also known as conjugate beamforming) is obtained. This precoding requires no CSI sharing between the antennas.

On the receiver side, the received radio signal is multiplied with the combining vector previously calculated in the uplink pilot phase. The output gives $K$ data streams. The processed streams are then combined with the data streams received from the shared bus and sent again on the shared bus to the next APU. More specifically, the $m$ th APU sums its received data streams to the input streams from APU $m-1$ consisting of combined signals from APUs $1, \ldots, m-1$, for one or more UEs. This cumulative signal is then outputted to APU $m+1$. The combination of signals is a simple per-stream addition operation.

The radio stripe system facilitates a flexible "cell-free" Massive MIMO deployment. While cellular APs are bulky, radio stripes enable invisible installation in existing construction elements as exemplified in Fig. 5b. The robustness is high since a few APUs can break with only a limited impact on the network. Moreover, a radio stripe deployment may integrate for example temperature sensors, microphones/speakers, or vibration sensors, and provide additional features such as fire alarms, burglar alarms, earthquake warning, indoor positioning, and climate monitoring and control.

\subsubsection{Front-haul and Back-haul Capacity}

While there is no need to share CSI between antenna elements in "cell-free" Massive MIMO, the CPUs must provide each APU with the data streams that its antenna elements will transmit. The data is delivered from the core network via the back-haul and then forwarded to the APU over the front-haul; see Fig. 5a. Similarly, the CPU will receive the cumulative signals from its the radio stripes over the front-haul and decode the data streams. The data streams will then be delivered to the core network over the back-haul.

The required capacity of the front-haul in a radio stripe is proportional to the number of simultaneous data streams that it could spatially multiplex at maximum network load. The required capacity of the back-haul of a CPU corresponds to the sum rate of the data streams that the radio stripes connected to the CPU will transmit/receive at maximum network load. The way to limit these capacity requirements is to constrain the number of UEs that can be served per AP (e.g., radio stripe) and CPU. To avoid creating cell boundaries, a user-centric perspective must be used when selecting which 
subset of APs that serve a particular UE, as illustrated in Fig. 1c.

Suppose a UE is alone in the network and all APs transmit to it with full power. Since the path-loss decays rapidly with the propagation distance, $95 \%$ of the received power will originate from a subset of the APs, called the 95\%-subset. When the ISD is large, as in a conventional cellular network, the 95\%-subset might only contain a handful of APs. As the ISD reduces (i.e., the number of antennas per $\mathrm{km}^{2}$ grows), there will be more APs in the $95 \%$-subset. This property has been utilized in [20] (see details in Section 1.4.1) to find the $95 \%$-subsets that maximize the energy efficiency of the network, taking into account the power consumption of back-haul signaling. The conclusion was that only $10-20 \%$ of the APs in the $1 \mathrm{~km}^{2}$ area surrounding the UE will belong to the $95 \%$-subset. In practice, the subset selection can be determined in the access phase, by measuring which APs that the UE receives a strong signal from.

\subsubsection{Synchronization}

To serve a UE by coherent joint transmission from multiple APs, the network infrastructure needs to be synchronized. The network might have an absolute time (phase) reference, but the APs are unsynchronized - they do not have access to this reference. This means that, effectively, the transmitter and receiver circuits of each AP have their own time references. The difference in time reference between the transmitter and receiver in a given AP represents the reciprocity (uplink-downlink) calibration error. The difference in, say, transmitter time reference, between any pair of APs represents the synchronization error between these two APs. A priori, all reciprocity and synchronization errors are unknown and needs to be synchronized at regular intervals.

Suppose the transmitter of $\mathrm{AP}_{i}$ has a clock bias of $t_{i}$ (i.e., its local time reference clock shows zero at absolute time $t_{i}$ ) and the receiver has a clock bias of $r_{i}$ (i.e., its clock shows zero at absolute time $r_{i}$ ). A simple synchronization protocol may then be implemented as follows:

1. At local time zero (absolute time $t_{1}$ ), $\mathrm{AP}_{1}$ transmits a known pulse. $\mathrm{AP}_{2}$ receives this pulse at time $t_{1}-r_{2}$, according to its clock, and timestamps it with $\delta_{12}=t_{1}-r_{2}$. Similarly, $\mathrm{AP}_{3}$ timestamps the pulse with its received local time, $\delta_{13}=t_{1}-r_{3}$.

2. At its local time zero, $\mathrm{AP}_{2}$ transmits a known pulse. $\mathrm{AP}_{1}$ timestamps the received pulse with its local reception time $\delta_{21}=t_{2}-r_{1} . \mathrm{AP}_{3}$ timestamps it with $\delta_{23}=t_{2}-r_{3}$. 
3. Finally, at its local time zero, $\mathrm{AP}_{3}$ transmits a known pulse. $\mathrm{AP}_{1}$ timestamps this received pulse with $\delta_{31}=t_{3}-r_{1}$. $\mathrm{AP}_{2}$ timestamps it with $\delta_{32}=t_{3}-r_{2}$.

The quantities $\delta_{i j}$ are known from the measurements. It is straightforward to see that $t_{1}, r_{1}, t_{2}, r_{2}, t_{3}, r_{3}$ cannot be obtained from $\delta_{i j}$ (the corresponding linear equation system is singular). However, the reciprocity and synchronization errors are easily recovered:

$$
\begin{aligned}
& t_{1}-r_{1}=\delta_{12}+\delta_{31}-\delta_{32} \\
& t_{2}-r_{2}=\delta_{21}+\delta_{32}-\delta_{31} \\
& t_{3}-r_{3}=\delta_{31}+\delta_{23}-\delta_{21} \\
& t_{1}-t_{2}=\delta_{13}-\delta_{23} \\
& t_{1}-t_{3}=\delta_{12}-\delta_{32} \\
& t_{2}-t_{3}=\delta_{21}-\delta_{31} .
\end{aligned}
$$

Next, consider two groups A and B, each group comprising three APs. The reciprocity errors within each group may be calibrated through the above-described procedure. The synchronization errors within each group may also be computed via that procedure, but each group will have an unknown remaining clock bias. This bias is the absolute time at which the transmitter's local clock of $\mathrm{AP}_{1}$ in the group shows zero. Suppose that at time zero, according to its local clock, $\mathrm{AP}_{1}$ in the first group transmits a known pulse. $\mathrm{AP}_{1}$ in the second group receives this pulse and timestamps it relative to its local clock; from these measurements the synchronization error between the two groups may be estimated. Extensions to groups larger than three nodes are straightforward and may enhance accuracy. The solution of an overdetermined equation system (e.g., via least squares) is then required.

This synchronization method can be straightforwardly applied in a differential manner. Consider measurements $\delta_{i j}$ taken at a first point in time at which the biases are $t_{1}, r_{1}, t_{2}, r_{2}, t_{3}, r_{3}$, and then measurements $\delta_{i j}^{\prime}$ taken at a second point in time at which the biases are $t_{1}^{\prime}, r_{1}^{\prime}, t_{2}^{\prime}, r_{2}^{\prime}, t_{3}^{\prime}, r_{3}^{\prime}$. The application of the above method to $\delta_{i j}^{\prime}-\delta_{i j}$ yields the evolution of clock biases, up to a drift that is common to the whole group.

The method outlined above can be applied to synchronize a "cell-free" network (e.g., consisting of radio stripes) over the air, without back-haul signaling, thus making the method scalable to large networks. 


\subsubsection{Power Control}

Power control is important to handle the near-far effect, and hence, protect any given UE from strong interference from other UEs. The power control is done at the CPU. The optimized power-control coefficients for the downlink and uplink are sent to the APs and UEs, respectively. Importantly, power control is performed once per large-scale fading realization which changes rather slowly. This implies that it needs to be updated only at infrequent intervals, i.e., a few times per second. In addition, the large-scale fading coefficients are essentially independent of frequency. Thus, power control in "cell-free" Massive MIMO is much simpler than the conventional wireless systems where the power control is performed once per small-scale fading realization.

Maximum ratio processing is the baseline scheme for "cell-free" Massive MIMO, since it can be implemented distributively [13]. The processed signals at the $l$ th AP and the $k$ th UE are represented in Fig. 6. In the downlink, at the $l$ th AP, the symbol intended for the $k$ th UE, $q_{k}$, is first weighted by $\hat{g}_{l k}^{*}$ and $\sqrt{\rho_{l k}}$, where $\hat{g}_{l k}$ is the estimate of the channel from AP $l$ to UE $k$ and $\rho_{l k}$ is the power-control coefficient. The weighted symbols of all $K$ UEs will be then combined and broadcasted to the UEs. In the uplink, at the $k$ th UE, the corresponding symbol $q_{k}$ is weighted by a power-control coefficient $\sqrt{\rho_{k}}$ before sending to the APs. In general, we aim at designing the power-control coefficients so that a given performance target (objective) is maximized under practical constraints. Depending on the applications, the objective can be max-min spectral efficiency, sum spectral efficiency, energy efficiency, or a combination thereof.

\section{Max-Min Fairness Power Control}

The goal of this policy is to deliver the same spectral efficiency to all UEs and making that spectral efficiency as large as possible, leading to a uniformly good UE service in the network. In practice, some UEs might have bad channels to all APs, due to unlucky pathloss and shadowing. With max-min fairness power control, substantial power has to be allocated to these UEs and the spectral efficiency might still be low, leading to bad service for everyone. Therefore, some deeply shadowed UEs need to be dropped from service to ensure a uniformly good service to all others. As in co-located Massive MIMO, effective and computationally tractable power control is one of the good properties of "cell-free" Massive MIMO. The max-min fairness power-control coefficients can be efficiently obtained by solving a sequence of linear programs for the uplink and a sequence of second-order cone programs 


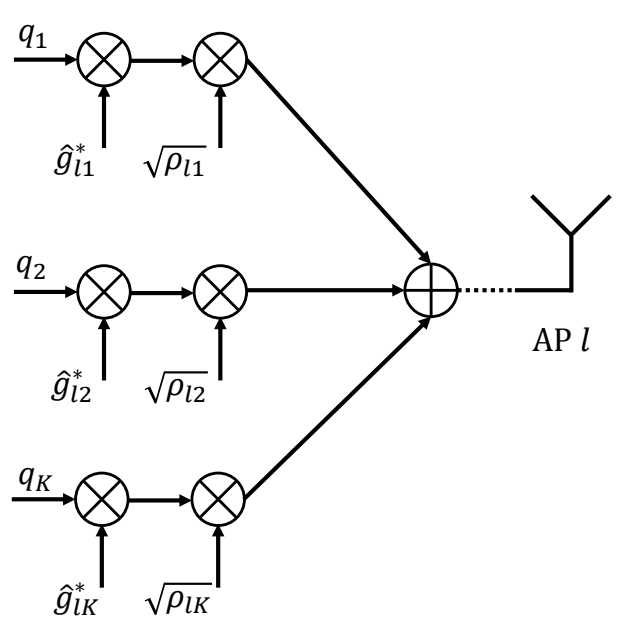

(a) Downlink.

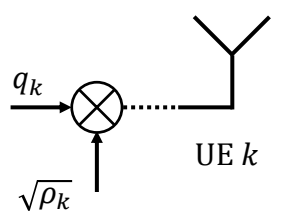

(b) Uplink.

Figure 6: Processed signals at the $l$ th AP (downlink) and the $k$ th UE (uplink) with maximum ratio precoding/combining.

(SOCPs) in the downlink [13].

\section{Power Control with User Prioritization}

The quality-of-service requirements are typically different among the UEs, which can be taken into account in the power control policy. For instance, the UEs with real-time services, such as video conferencing and online gaming, can be given higher transmission priority (according to their QoS requirements) than the UEs with non-real-time services such as file downloading and email services. Furthermore, UEs willing to pay for extra service could be allocated a higher guaranteed quality of service than what the typical UE gets. The max-min fairness power control can be extended to consider weighted spectral efficiencies, where each UE has an individual weight that represents its priority. Minimum spectral efficiency constraints can be also included. Similar optimization procedures can be used as for the unweighted problem.

For these cases, power control with user prioritization can be exploited via a direct extension of max-min fairness power control to a max-min weighted spectral efficiency problem. In the max-min weighted spectral efficiency problem, the $K$ users are weighted according to priority, i.e., a user with higher priority will be weighted with a smaller factor. 


\section{Power Control with AP Selection}

Since the APs and UEs are located over a large area, many APs are very far away from each given UE and will not contribute much to its performance. Only the subset of closest APs should serve the UE, which could be the 95\%-subset defined before. Mathematically, only the APs that serve a UE is allowed to have non-zero power-control coefficients related to that UE.

\subsubsection{Pilot Assignment}

To reduce pilot contamination, efficient pilot assignment schemes should be exploited. Below is a list of common pilot assignment schemes which can be applied to "cell-free" Massive MIMO:

- Random pilot assignment: Each UE is randomly assigned one pilot sequence from the predefined set of pilot sequences. Typically, the pilot sequences in this set are mutually orthogonal. This method is easy to implement, but there is a high probability that some closely located UEs will use the same pilot, leading to high pilot contamination and bad performance.

- Greedy pilot assignment: The $K$ UEs are first assigned pilot sequences through the random pilot assignment method. Then the assignment is iteratively improved with respect to some utility, such as maximizing the spectral efficiency for the worst UE (the UE with lowest spectral efficiency or the largest amount of pilot contamination). The algorithm needs to proceed iteratively.

- Brute-force assignment: A search over all possible pilot sequences can be performed to maximize a utility such as the max-min spectral efficiency or sum spectral efficiency. This method is optimal but the complexity grows exponentially with the number of UEs.

To achieve good network performance, pilot assignment and power control can be performed jointly.

\subsection{Performance of "cell-free" Massive MIMO}

The radio stripe system, described earlier, represents a cost-efficient build practice of a ubiquitous "cell-free" Massive MIMO system. Furthermore, it might be an ideal solution in terms of flexibility and scalability of the deployment, and to provide coverage to environments where large and visible 
installations, typical of centralized network architectures, are not admissible either because of regulation or space constraints.

In this regard, we next analyze the performance provided by a radio-stripe system in two case studies of practical interest: $(i)$ industrial indoor scenario, and (ii) piazza scenario (outdoor).

\subsubsection{Industrial Indoor Scenario}

Nowadays, wireless communication plays a central role in the industrial production process. Ubiquitous coverage, low latency, ultra-reliable communication, and resilience are key for wireless communications in a factory environment. In this respect, "cell-free" Massive MIMO, with its flexible distributed architecture, with its macro-diversity gain and inherent ability to suppress interference, is suitable to cope with the challenging industrial indoor scenario. Also, a radio stripe deployment may integrate additional sensors/actuators such as temperature sensors, microphones, miniature speakers, vibration sensors, etc., and provide additional important features, e.g., fire alarm, burglar alarm, earthquake warning, indoor positioning, climate monitoring and control.

In our simulations, we consider an industrial environment described as in [21]: a building 7-8 $\mathrm{m}$ high with metal ceiling, and concrete floors and walls. Industrial inventory mainly consists of metal machinery. The radio stripes are deployed in an area of $100 \times 100$ meters in such a way that 400 APs shape a $20 \times 20$ regular grid, as shown in Fig. 7a (left). Hence, the end-most antennas are $5 \mathrm{~m}$ apart. They are placed at $6 \mathrm{~m}$ above ground level, while the UE antenna height is $2 \mathrm{~m}$. The carrier frequency that we consider is $5200 \mathrm{MHz}$, which is within the frequency band $5150-5825 \mathrm{MHz}$ adopted for application of indoor industrial wireless communications. Hence, the antenna element size, in the case $\lambda / 2$ antenna is implemented, is $2.8 \mathrm{~cm}$.

The performance, in terms of per-user spectral efficiency and the impact of power control is shown in Fig. 7a (right). We consider $K=20$ uniformly distributed UEs, mutually orthogonal pilots, and four different downlink power control settings, assuming a maximum per-AP radiated power of 200 $\mathrm{mW}$ :

1. CD-FPT: Channel-dependent full power transmission. All APs transmit with full power and the power control coefficients for a given AP $l$ are the same for all $k=1, \ldots, K$. The power control coefficient between AP $l$ and UE $k$ is given by $\rho_{l k}=\left(\sum_{k^{\prime}=1}^{K} \gamma_{l k^{\prime}}\right)^{-1}$, where $\gamma_{l k^{\prime}}$ is the variance of the corresponding channel estimate $\hat{g}_{l k^{\prime}}$; 
2. MMF: Max-min fairness power control. All the APs are involved in coherently serving a given UE. The power control coefficients are chosen to maximize the minimum spectral efficiency of the network, as described in detail in [13, Section IV-B].

3. MMF-RPB AP selection [20]: Max-min fairness power control with received-power-based AP selection. Only a subset of APs serves a given UE $k$. The subset consists of the APs that contribute at least $\alpha \%$ (e.g., $95 \%$, as described before) of the power assigned to UE $k$. Mathematically,

$$
\sum_{l=1}^{\left|\mathcal{A}_{k}\right|} \frac{\varrho_{l k}}{\sum_{j=1}^{L} \sqrt{\rho_{j k}} \gamma_{j k}} \geq \alpha \%,
$$

where $\left|\mathcal{A}_{k}\right|$ is the cardinality of the user- $k$-specific AP subset, and $\left\{\varrho_{1 k}, \ldots, \varrho_{L k}\right\}$ is the set of the coefficients $\varrho_{l k} \triangleq \sqrt{\rho_{l k}} \gamma_{l k}$ sorted in descending order.

4. MMF-CQB AP selection [20]: Max-min fairness power control with channel-quality-based AP selection. This method selects the APs with the best channel quality (largest large-scale fading coefficient) towards UE $k$ as follows

$$
\sum_{l=1}^{\left|\mathcal{A}_{k}\right|} \frac{\bar{\beta}_{l k}}{\sum_{j=1}^{L} \beta_{j k}} \geq \alpha \%,
$$

where $\beta_{j k}$ is the large-scale fading coefficient of the channel between the $j$ th $\mathrm{AP}$ and the $k$ th UE, and $\left\{\bar{\beta}_{1 k}, \ldots, \bar{\beta}_{L k}\right\}$ is the set of the large-scale fading coefficients sorted in descending order.

Max-min power control doubles the 95\%-likely spectral efficiency compared to the baseline CD-FPT case. Thanks to optimal power control, the radio-stripe system can guarantee to each UE almost $4.5 \mathrm{bit} / \mathrm{s} / \mathrm{Hz}$. The performance with AP selection is also exploited (dashed and dashed dotted lines). We can see that the spectral efficiency reduction is minor if the RPB AP selection strategy is used, while the CQB criterion leads to a $20 \%$ reduction. The performance gap is attributable to the cardinality of the corresponding AP subsets; on average, CQB uses $17 \%$ of the APs and RPB uses $42 \%$ of the APs. RPB AP selection strategy takes into account the power allocated to a given UE after max-min power control. The effect of performing max-min power control is basically to include more APs in the $95 \%$-subset, as it sets the power control coefficients to compensate the path loss and give the same spectral efficiency to all the UEs. 

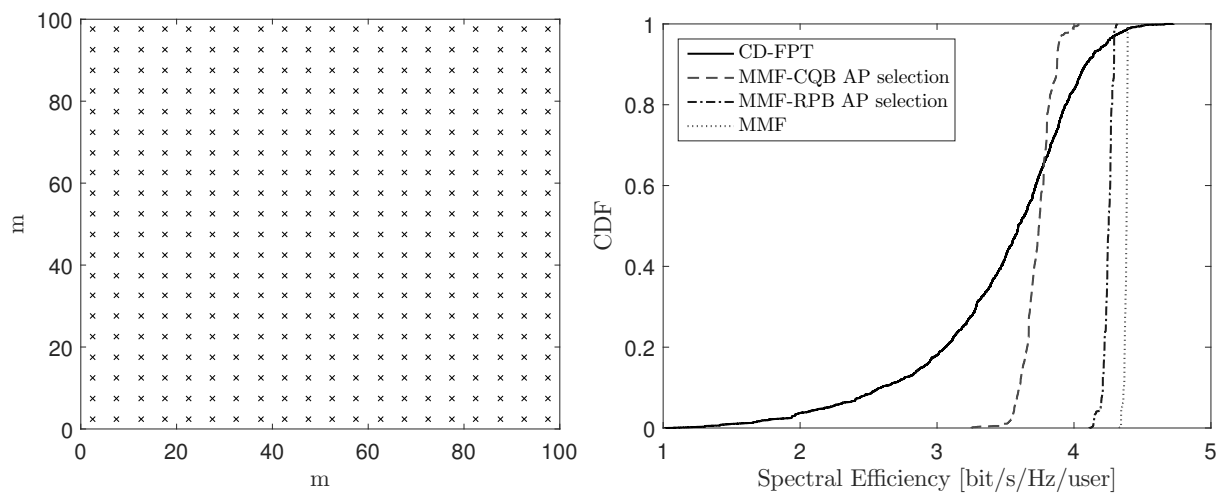

(a) Industrial indoor scenario. We use the one-slope path loss model defined by $\operatorname{PL}(d)=$ $\mathrm{PL}\left(d_{0}\right)+10 n \log _{10}\left(d / d_{0}\right)$, where $d$ is the distance in meters between the transmitter and the receiver, $\mathrm{PL}\left(d_{0}\right)$ is the path loss in $\mathrm{dB}$ measured at a reference distance $d_{0}$ in meters, and $n$ is the dimensionless path loss exponent. The shadowing follows a lognormal distribution with median equal to $0 \mathrm{~dB}$ and standard deviation $\sigma \mathrm{dB}$. According to [21], a large-scale fading topography that includes line-of-sight and obstructed line-of-sight paths with both light and heavy surrounding clutter, with $d_{0}=15 \mathrm{~m}$, is characterized by: $\operatorname{PL}\left(d_{0}\right)=70.28, n=2.59$, and $\sigma=6.09$. The small-scale fading is modeled by independent Rayleigh fading.
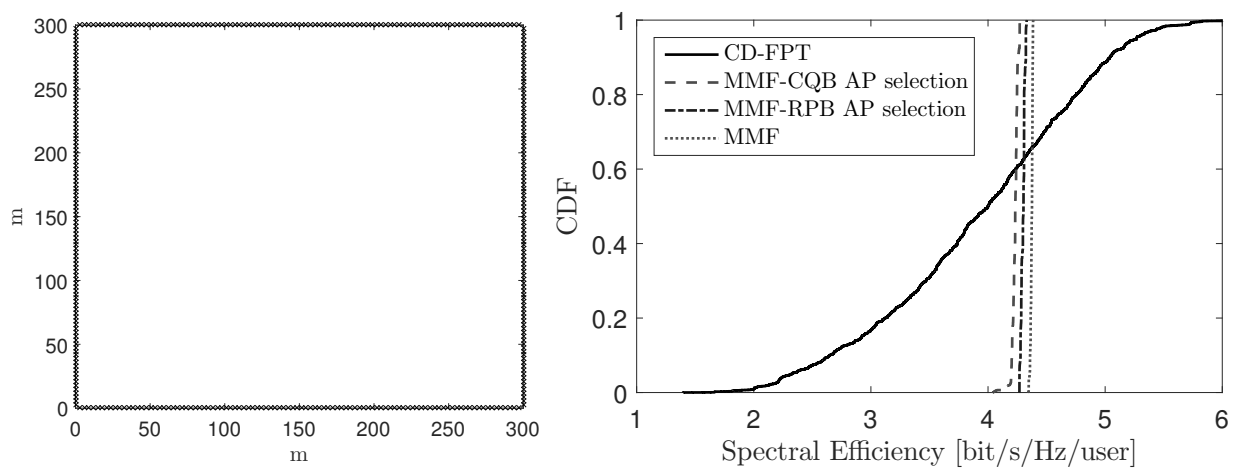

(b) Piazza scenario. The large-scale fading is modeled as in [13], assuming uncorrelated shadow fading. The small-scale fading follows i.i.d. Rayleigh distribution.

Figure 7: Left figures illustrate the APs deployments. On the right, the CDF for the per-user spectral efficiency, as defined in [13, Section III-B]. We choose $L=400$ and $K=20$. To simulate no cell boundaries, we wrap the simulation area around with eight twin neighbor areas. 


\subsubsection{Piazza Scenario}

Installations causing a big visual impact on the environment can be prohibited in areas like piazzas and historic places. In such a scenario, a radio-stripe system can provide all the advantages previously described with an unobtrusive deployment. We consider a radio-stripe system that gives coverage to a square area of $300 \times 300$ meters (e.g., historic piazza). The radio stripes are placed along the perimeter of the square at $9 \mathrm{~m}$ height, supposing they are on the facade of the buildings surrounding the piazza. At the carrier frequency of $2 \mathrm{GHz}$, the $\lambda / 2$ antenna element size is $7.5 \mathrm{~cm}$. Assuming a total of 400 APs deployed in this scenario, that is 100 APs per square side, the end-most APs are $3 \mathrm{~m}$ apart. We consider 20 uniformly distributed UEs, mutually orthogonal pilots, and the same power control profiles as before. Finally, the maximum per-APs radiated power is set to $400 \mathrm{~mW}$ to deal with such a wide coverage area. The numerical results are shown in Fig. 7b.

Under these assumptions, the radio-stripe system with max-min power control can provide a per-user spectral efficiency around $4.5 \mathrm{bit} / \mathrm{s} / \mathrm{Hz}$, doubling the performance compared to the baseline CD-FPT. Due to the AP deployment symmetry, the AP selection strategies perform almost equally well; CQB and RPB select around $1 / 3$ of the APs on average. The performance gap with respect the case with no AP selection is negligible, thus $2 / 3$ of the APs can be left out in the transmission towards a given UE.

\subsection{Research Directions}

The "cell-free" topology brings the best of two worlds: the macro-diversity from distributing many APs and the interference cancelation from cellular Massive MIMO. While this article has outlined the basic processing and implementation concepts, many open issues remain, ranging from communication theory to measurements and engineering efforts:

- Power control: While (weighted) max-min power-control is computationally tractable and provides uniform quality of service, it does not take actual traffic patterns into account. New power control algorithms are needed to balance fairness, latency, and network throughput, while permitting a distributed implementation.

- Distributed signal processing: Maximum ratio precoding/detection and synchronization can be distributed, as described earlier, but the data encoding/decoding must be carried out at one or multiple CPUs. The distribution of such signal processing tasks over the network is 
non-trivial, when looking for a good tradeoff between high rates and limited back-haul signaling.

- Resource allocation and broadcasting: Scheduling, pilot allocation, system information broadcast, and random access are basic functionalities that traditionally rely on a cellular architecture. New algorithms and protocols are needed for these tasks in "cell-free" networks.

- Channel modeling: The performance analysis of "cell-free" networks have primarily considered Rayleigh fading channels. Practical channels are likely to contain a mix of line-of-sight and non-line-of-sight paths, and will likely differ substantially depending on the carrier frequency. Dedicated channel measurements followed by refined channel modeling are necessary to better understand the channel characteristics and fine-tune resource allocation algorithms.

- DL channel estimation: DL channel estimates, needed for data decoding, can either be obtained from DL pilots, which increases the pilot overhead, or by blind estimation techniques that uses the DL data. Dedicated algorithms for this estimation are needed.

- Compliance with existing standards: The 5G standard is intended to be forward-compatible and only relies on cell-identities for the basic functionalities. It is likely that "cell-free" data transmission can be implemented in 5G, but further work in standardization and conceptual development is needed.

- Prototype development: The step from a promising communication concept to a practical network requires substantial prototyping. The first working "cell-free" prototype may be pCell, where [22] describes a setup with 32 APs serving 16 UEs. Since every AP in a "cell-free" network has low cost and footprint, prototyping can be carried out using rather simple components. One can begin by demonstrating the synchronization and joint processing capabilities with a small number of APs in a limited area, and then continue with more APs and larger coverage area. 



\section{Chapter 2}

\section{Contributions of the Thesis}

This thesis focuses on some signal processing aspects of cell-free massive MIMO. In Paper A, we investigate the benefits of including downlink pilots in the cell-free massive MIMO TDD protocol. To improve the system performance, we jointly optimize the downlink power allocation and the pilot assignment. A utility-based method for orthogonal downlink pilot assignment is proposed in Paper B. Such a method aims to save resources by allocating the downlink pilots based on the user's real need to estimate the downlink channel. In Paper C and Paper D we investigate further fully distributed and scalable precoding schemes for cell-free massive MIMO systems. We propose, respectively, a normalized conjugate beamforming that has the merit of boosting the channel hardening and reducing the beamforming gain uncertainty; a full-pilot zero-forcing scheme that, by exploiting multi-antenna APs, suppresses inter-user interference and guarantees larger spectral efficiency.

\subsection{Introduction of the Thesis}

Chapter 1 of this thesis is based on material from the following paper:

\section{Ubiquitous Cell-Free Massive MIMO Communications}

Authored by: Giovanni Interdonato, Emil Björnson, Hien Quoc Ngo, Pål Frenger, and Erik G. Larsson

Submitted to: IEEE Communications Magazine, April 2018 


\subsection{Papers Included in the Thesis}

\section{Paper A: Downlink Training in Cell-Free Massive MIMO: A Blessing in Disguise}

Authored by: Giovanni Interdonato, Hien Quoc Ngo, Pål Frenger and Erik G. Larsson

Under preparation for submission to: journal to be defined.

Abstract: Cell-free Massive MIMO (multiple-input multiple-output) refers to a distributed Massive MIMO system where all the access points (APs) cooperate to coherently serve all the user equipments (UEs), suppress intercell interference and mitigate the multiuser interference. Recent works $[16,17]$ demonstrated that, unlike co-located Massive MIMO, the channel hardening is, in general, less pronounced in cell-free Massive MIMO, thus there is much to benefit from estimating the downlink channel. In this study, we investigate the gain introduced by the downlink beamforming training [23], extending the analysis in [17] to non-orthogonal uplink and downlink pilots. Assuming single-antenna APs, conjugate beamforming and independent Rayleigh fading channel, we derive a closed-form expression for the per-user achievable downlink rate that addresses channel estimation errors and pilot contamination both at the AP and UE side. The performance evaluation also includes max-min fairness power control, greedy pilot assignment methods, and a comparison between achievable rates obtained from different capacitybounding techniques. We also investigate the impact of the downlink pilots in cell-free Massive MIMO with user-centric approach. Numerical results show that downlink beamforming training, although increases pilot overhead and introduces additional pilot contamination, improves significantly the achievable downlink rate. Even for large number of APs, it is not fully efficient for the UE relying on the statistical channel state information for data decoding. 


\section{Paper B: Utility-based Downlink Pilot Assignment in Cell-Free Massive MIMO}

Authored by: Giovanni Interdonato, Hien Quoc Ngo, Pål Frenger and Erik G. Larsson

Published in Proceedings of: 2018 22nd International ITG Workshop on Smart Antennas (WSA), pp. 1-8, March 2018.

Abstract: We propose a strategy for orthogonal downlink pilot assignment in cell-free massive MIMO (multiple-input multiple-output) that exploits knowledge of the channel state information, the channel hardening degree at each user, and the mobility conditions for the users. These elements, properly combined together, are used to define a user pilot utility metric, which measures the user's real need of a downlink pilot for efficient data decoding. The proposed strategy consists in assigning orthogonal downlink pilots only to the users having a pilot utility metric exceeding a predetermined threshold. Instead, users that are not assigned with an orthogonal downlink pilot decode the data by using the statistical channel state information. The utility-based approach guarantees higher downlink net sum throughput, better support both for high-speed users and shorter coherent intervals than prior art approaches.

\section{Paper C: On the Performance of Cell-Free Massive MIMO with Short-Term Power Constraints}

Authored by: Giovanni Interdonato, Hien Quoc Ngo, Erik G. Larsson and Pål Frenger

Published in Proceedings of: 2016 IEEE 21st International Workshop on Computer Aided Modelling and Design of Communication Links and Networks (CAMAD), pp. 225-230, October 2016.

Abstract: In this paper we consider a time-division duplex cell-free massive multiple-input multiple-output (MIMO) system where many distributed access points (APs) simultaneously serve many users. A normalized conjugate beamforming scheme, which satisfies short-term average power constraints at the APs, is proposed and analyzed taking into account the effect of imperfect channel information. We derive an approximate closed-form expression for the per-user achievable downlink rate of this scheme. We also provide, analytically and numerically, a performance comparison between the normalized conjugate beamforming and the conventional conjugate beamforming scheme in [13] (which satisfies long-term average power constraints). Normalized conjugate beamforming scheme reduces the beamforming gain uncertainty, which comes from the users' lack of the channel state information knowledge, and hence, it 
improves the achievable downlink rate compared to the conventional conjugate beamforming scheme.

\section{Paper D: Downlink Spectral Efficiency of Cell-Free Massive MIMO with Full-Pilot Zero-Forcing}

Authored by: Giovanni Interdonato, Marcus Karlsson, Emil Björnson and Erik G. Larsson

Submitted to: 2018 6th IEEE Global Conference on Signal and Information Processing (GlobalSIP).

Abstract: Cell-free Massive multiple-input multiple-output (MIMO) ensures ubiquitous communication at high spectral efficiency (SE) thanks to increased macro-diversity as compared cellular communications. However, system scalability and performance are limited by fronthauling traffic and interference. Unlike conventional precoding schemes that only suppress intra-cell interference, full-pilot zero-forcing (fpZF), introduced in [24], actively suppresses also inter-cell interference, without sharing channel state information (CSI) among the access points (APs). In this study, we derive a new closed-form expression for the downlink (DL) SE of a cell-free Massive MIMO system with multi-antenna APs and fpZF precoding, under imperfect CSI and pilot contamination. The analysis also includes max-min fairness DL power optimization. Numerical results show that fpZF significantly outperforms maximum ratio transmission scheme, without increasing the fronthauling overhead, as long as the system is sufficiently distributed.

\subsection{Papers Not Included in the Thesis}

\section{Paper E: How Much Do Downlink Pilots Improve Cell-Free Massive MIMO?}

Authored by: Giovanni Interdonato, Hien Quoc Ngo, Erik G. Larsson and Pål Frenger

Published in Proceedings of: 2016 IEEE Global Communications Conference (GLOBECOM), pp. 1-7, December 2016.

This paper contains preliminary results of Paper A. 


\section{Chapter 3}

\section{Conclusions}

Cell-free massive multiple-input multiple-output (MIMO) ensures ubiquitous communication at high spectral efficiency (SE) thanks to increased macrodiversity as compared cellular communications. A conventional cell-free massive MIMO system consists in single-antenna access points (APs) implementing maximum ratio precoding, and single-antenna users implementing maximum ratio combining. The users perform data decoding relying on the channel hardening phenomenon, by exploiting the knowledge of the statistical channel state information (CSI).

In this thesis, we demonstrated that letting the users estimate the downlink channel, via downlink pilots, can improve considerably the achievable downlink rate. The benefits introduced by the downlink pilots are substantial, justifying the additional pilot overhead and interference from pilot contamination. This holds even for large number of APs where the channel hardening degree is larger, and for relatively short coherence interval where the pilot overhead becomes more significant. Larger performance improvements can be obtained by jointly optimizing the downlink power allocation and the uplink/downlink pilot assignment. Max-min fairness power control and greedy pilot assignment algorithms provides uniform good quality of service throughout the network, by mitigating inter-user interference and pilot contamination. Downlink pilots can be allocated properly by exploiting the statistical CSI knowledge at the CPU. The utility-based downlink pilot assignment method, proposed in this thesis, allows to increase the sum throughput, and saves radio resources when it comes with mutually orthogonal pilot allocation.

We also proposed two fully distributed and scalable precoding schemes for cell-free massive MIMO. Normalized conjugate beamforming performs only a phase-shift of the data signal and can be implemented by single-antenna 
APs. This scheme boosts the channel hardening at the users, by reducing the beamforming gain uncertainty, which comes from the users' lack of instantaneous CSI. Hence, it improves the achievable downlink rate compared to conventional conjugate beamforming. Full-pilot zero-forcing must be implemented by multi-antenna APs. Unlike conventional zero-forcing, fullpilot zero-forcing actively suppresses inter-user interference without sharing CSI between the APs and the CPU. It significantly outperforms conjugate beamforming scheme, without increasing the fronthauling overhead, as long as the system is sufficiently distributed.

Regarding practical deployment issues, the proposed radio stripe system constitutes a flexible cost-efficient architecture for cell-free massive MIMO deployment. Radio stripes enable compute-and-forward signal processing making the system fully scalable. Also, while cellular APs are bulky, radio stripes allow invisible installation in existing construction elements. 


\section{Chapter 4}

\section{Future Work}

Cell-free massive MIMO is a relatively new topic. It is plenty of directions to exploit for future work. Blind channel estimation methods, to let the users estimate the downlink channel from the data symbols, need to be investigated to give the final answer whether we can do without the downlink pilots in cell-free massive MIMO. Also, a performance comparison between normalized conjugate beamforming and conjugate beamforming scheme with downlink pilots, at the respective optimal operational points, is certainly interesting. The analysis for the utility-based downlink pilot assignment strategy can be conveniently extended to non-orthogonal pilots (and pilot reuse).

While (weighted) max-min power-control is computationally tractable and provides uniform quality of service, the calculation of the power control coefficients is still centralized. New power control algorithms are needed to allow fully distributed and scalable implementation, while providing good (even though non-optimal) performance. Designing a system as much distributed and scalable as possible is key. Maximum ratio precoding, full-pilot zero-forcing and synchronization can be distributed, as described earlier, but the data encoding/decoding must be carried out at one or multiple CPUs. The distribution of such signal processing tasks over the network is non-trivial, when looking for a good tradeoff between high rates and limited back-haul signaling.

The performance analysis of "cell-free" networks have primarily considered Rayleigh fading channels. Practical channels are likely to contain a mix of line-of-sight and non-line-of-sight paths, and will likely differ substantially depending on the carrier frequency. New analyses and performance evaluations under refined channel modeling are necessary to better understand the potential of cell-free massive MIMO. 



\section{Bibliography}

[1] W. R. Young, "Advanced mobile phone service: Introduction, background, and objectives," Bell System Technical Journal, vol. 58, no. 1, pp. 1-14, 1979.

[2] J. G. Andrews, X. Zhang, G. D. Durgin, and A. K. Gupta, "Are we approaching the fundamental limits of wireless network densification?" IEEE Commun. Mag., vol. 54, no. 10, pp. 184-190, Oct. 2016.

[3] S. Zhou, M. Zhao, X. Xu, J. Wang, and Y. Yao, "Distributed wireless communication system: a new architecture for future public wireless access," IEEE Commun. Mag., vol. 41, no. 3, pp. 108-113, Mar. 2003.

[4] T. L. Marzetta, "Noncooperative cellular wireless with unlimited numbers of base station antennas," IEEE Trans. Wireless Commun., vol. 9, no. 11, pp. 3590-3600, Nov. 2010.

[5] T. L. Marzetta, E. G. Larsson, H. Yang, and H. Q. Ngo, Fundamentals of Massive MIMO. Cambridge University Press, 2016.

[6] J. G. Andrews, S. Buzzi, W. Choi, S. V. Hanly, A. Lozano, A. C. K. Soong, and J. C. Zhang, "What will 5G be?" IEEE J. Sel. Areas Commun., vol. 32, no. 6, pp. 1065-1082, 2014.

[7] S. Shamai and B. M. Zaidel, "Enhancing the cellular downlink capacity via co-processing at the transmitting end," in Proc. IEEE VTC-Spring, vol. 3, 2001, pp. 1745-1749.

[8] S. Venkatesan, A. Lozano, and R. Valenzuela, "Network MIMO: Overcoming intercell interference in indoor wireless systems," in Proc. IEEE ACSSC, 2007, pp. 83-87.

[9] G. Caire, S. Ramprashad, and H. Papadopoulos, "Rethinking network MIMO: Cost of CSIT, performance analysis, and architecture comparisons," in Proc. IEEE ITA, 2010. 
[10] M. Boldi, A. Tölli, M. Olsson, E. Hardouin, T. Svensson, F. Boccardi, L. Thiele, and V. Jungnickel, "Coordinated multipoint (CoMP) systems," in Mobile and Wireless Communications for IMT-Advanced and Beyond, A. Osseiran, J. Monserrat, and W. Mohr, Eds. Wiley, 2011, pp. 121-155.

[11] A. Osseiran, J. F. Monserrat, and P. Marsch, 5G Mobile and Wireless Communications Technology. Cambridge University Press, 2016, ch. 9, Coordinated Multi-Point Transmission in 5G.

[12] D. Gesbert, S. Hanly, H. Huang, S. Shamai, O. Simeone, and W. Yu, "Multi-cell MIMO cooperative networks: A new look at interference," IEEE J. Sel. Areas Commun., vol. 28, no. 9, pp. 1380-1408, Dec. 2010.

[13] H. Q. Ngo, A. Ashikhmin, H. Yang, E. G. Larsson, and T. L. Marzetta, "Cell-free Massive MIMO versus small cells," IEEE Trans. Wireless Commun., vol. 16, no. 3, pp. 1834-1850, Mar. 2017.

[14] E. Nayebi, A. Ashikhmin, T. L. Marzetta, H. Yang, and B. D. Rao, "Precoding and power optimization in cell-free Massive MIMO systems," IEEE Trans. Wireless Commun., vol. 16, no. 7, pp. 4445-4459, Jul. 2017.

[15] E. Björnson, R. Zakhour, D. Gesbert, and B. Ottersten, "Cooperative multicell precoding: Rate region characterization and distributed strategies with instantaneous and statistical CSI," IEEE Trans. Signal Process., vol. 58, no. 8, pp. 4298-4310, Aug. 2010.

[16] Z. Chen and E. Björnson, "Channel hardening and favorable propagation in cell-free Massive MIMO with stochastic geometry," IEEE Trans. Commun., pp. 1-1, 2018.

[17] G. Interdonato, H. Q. Ngo, E. G. Larsson, and P. Frenger, "How much do downlink pilots improve cell-free Massive MIMO?" in 2016 IEEE Global Commun. Conf. (GLOBECOM), 2016, pp. 1-7.

[18] J. Jose, A. Ashikhmin, T. L. Marzetta, and S. Vishwanath, "Pilot contamination and precoding in multi-cell TDD systems," IEEE Trans. Commun., vol. 10, no. 8, pp. 2640-2651, Aug. 2011.

[19] P. Frenger, J. Hederen, M. Hessler, and G. Interdonato, "Improved antenna arrangement for distributed massive MIMO," WO patent application 2018103897 , Jan., 2017. [Online]. Available: patentscope.wipo.int/search/en/WO2018103897 
[20] H. Q. Ngo, L. N. Tran, T. Q. Duong, M. Matthaiou, and E. G. Larsson, "On the total energy efficiency of cell-free Massive MIMO," IEEE Trans. Green Commun. Netw., vol. 2, no. 1, pp. 25-39, Mar. 2018.

[21] E. Tanghe, W. Joseph, L. Verloock, L. Martens, H. Capoen, K. V. Herwegen, and W. Vantomme, "The industrial indoor channel: largescale and temporal fading at 900, 2400, and $5200 \mathrm{MHz}, "$ IEEE Trans. Wireless Commun., vol. 7, no. 7, pp. 2740-2751, Jul. 2008.

[22] A. Forenza, S. Perlman, F. Saibi, M. D. Dio, R. van der Laan, and G. Caire, "Achieving large multiplexing gain in distributed antenna systems via cooperation with pCell technology," in 49th Asilomar Conf. Signals, Syst., Comput., 2015, pp. 286-293.

[23] H. Q. Ngo, E. G. Larsson, and T. L. Marzetta, "Massive MU-MIMO downlink TDD systems with linear precoding and downlink pilots," in Proc. Allerton Conf. Commun., Control and Comp., 2013, pp. 293-298.

[24] E. Björnson, E. G. Larsson, and M. Debbah, "Massive MIMO for maximal spectral efficiency: How many users and pilots should be allocated?" IEEE Trans. Wireless Commun., vol. 15, no. 2, pp. 12931308, Feb. 2016. 


\section{Papers}

The papers associated with this thesis have been removed for copyright reasons. For more details about these see:

http://urn.kb.se/resolve?urn=urn:nbn:se:liu:diva-151026 


\section{Other Recently Published Theses From \\ The Division of Communication Systems \\ Department of Electrical Engineering (ISY) \\ Linköping University, Sweden}

Marcus Karlsson, Blind Massive MIMO Base Stations: Downlink Transmission and Jamming, Linköping Studies in Science and Technology. Dissertations, No. 1950, 2018.

Daniel Verenzuela, Analysis of Alternative Massive MIMO Designs: Superimpsed Pilots and Mixed-ADCs, Linköping Studies in Science and Technology. Licentiate Thesis, No. 1803, 2018.

Hei Victor Cheng, Optimizing Massive MIMO: Precoder Design and Power Allocation, Linköping Studies in Science and Technology. Dissertations, No. 1929, 2018.

Christopher Mollén, High-End Performance with Low-End Hardware: Analysis of Massive MIMO Base Station Transceivers, Linköping Studies in Science and Technology. Dissertations, No. 1896, 2017.

Trịnh Văn Chiến, Resource Allocation for Max-Min Fairness in Multi-Cell Massive MIMO, Linköping Studies in Science and Technology. Licentiate Thesis, No. 1797, 2017.

Antonios Pitarokoilis, Phase Noise and Wideband Transmission in Massive MIMO, Linköping Studies in Science and Technology. Dissertations, No. 1756, 2016.

Anu Kalidas M. Pillai, Signal Reconstruction Algorithms for Time-Interleaved ADCs, Linköping Studies in Science and Technology. Dissertations, No. 1672, 2015.

Ngô Quốc Hiển, Massive MIMO: Fundamentals and System Designs, Linköping Studies in Science and Technology. Dissertations, No. 1642, 2015.

Mirsad Čirkić, Efficient MIMO Detection Methods, Linköping Studies in Science and Technology. Dissertations, No. 1570, 2014.

Reza Moosavi, Improving the Efficiency of Control Signaling in Wireless Multiple Access Systems, Linköping Studies in Science and Technology. Dissertations, No. 1556, 2014.

Johannes Lindblom, The MISO Interference Channel as a Model for Non-Orthogonal Spectrum Sharing, Linköping Studies in Science and Technology. Dissertations, No. $1555,2014$.

Tumula V. K. Chaitanya, HARQ Systems: Resource Allocation, Feedback Error Protection, and Bits-to-Symbol Mappings, Linköping Studies in Science and Technology. Dissertations, No. 1555, 2013. 